\title{
EXPERIMENTAL STUDIES ON THE RHEOLOGICAL PROPERTIES OF COBALT FERRITE NANOFLUIDS
}

\section{DUONG HONG QUYEN \& HOANG THI KIEU NGUYEN}

Hanoi University of Science and Technology,No 1 Dai Co Viet, Hanoi, Vietnam

\begin{abstract}
Cobalt ferrite $\left(\mathrm{CoFe}_{2} \mathrm{O}_{4}\right)$ nanoparticles and corresponding cobalt ferrite nanofluids are one of the most recently explored magnetic particles by their improved magnetic properties in comparison with the conventional ferrofluids. In this paper, the viscosity behaviors of two types of cobalt ferrite nanofluids were studied. One of these was based on $\mathrm{CoFe}_{2} \mathrm{O}_{4}$ ferrimagnetic particles with an average diameter of $10.7 \mathrm{~nm}$, whereas the other was based on $\mathrm{CoFe}_{2} \mathrm{O}_{4}$ superparamagnetic particles of 5.8 $\mathrm{nm}$ in diameter. The dynamic viscosities of these nanofluids were measured as the shear rate dependence under the magnetic field of various intensities, using a rotating rheometer. The cobalt ferrite fluids exhibit a yield shear-thinning behavior with and without magnetic field application. The magnetoviscous effects are somewhat lower than those of the standard iron oxide ferrofluids. The more substantial magnetorheological effect in the case of nanofluids based on super para magnetic particles is observed. This result is attributed to the difference in number density and magnetic properties of both fluids. The concentration-dependent viscosity of the cobalt ferritefluids was considered. The experimental data indicate that the viscosity is enhanced with the increase of magnetic field strength and nanoparticle weight fraction. At the shear rate high enough, the viscosity of all the fluids tends to saturate at the concentrations higher than $5 \%$.
\end{abstract}

KEYWORDS: Cobalt Ferrite; Magnetic Nanofluids; Rheological Property \& Magnetoviscous

Received: Mar 19, 2020; Accepted: Apr 10, 2020; Published: May 22, 2020; Paper Id.: IJMPERDJUN202063

\section{INTRODUCTION}

Magnetic nanoparticles have shown tremendous potential in various applications because of their unique material properties. Among different magnetic nanomaterials, magnetite and maghemite are widely studied by their excellent properties for biomedical applications. However, a challenge to be addressed regarding iron oxide nanoparticles is their poor magnetic properties at small sizes and under physiological conditions (Srinivasan, 2018). Considering a need for alternative materials that exhibit improved magnetic characteristics, Cobalt Ferrite NanoParticles (CFNPs) are attractive for their unique properties (Sabah H. Sabeeh, 2017). CFNPs is often wellknown as a ferrimagnetic material with moderatemagnetization $(80 \mathrm{emu} / \mathrm{g})$ and strong magnetocrystalline anisotropy (K1 $\approx 2 \times 106 \mathrm{erg} / \mathrm{cm} 3$ )(Shahjuee, 2017). At the nanoscale size less than $10 \mathrm{~nm}$, cobalt ferrite may show excellent superparamagnetic behaviors, for example, high specific absorption rates for magnetic hyperthermia and excellent relaxation time ratios for MRI(Sharifi, 2012) (Amiri \& Shokrollahi, 2013). Most of the current researches has been focussed on the modification of CFNP properties through changing preparation methods, reaction conditions, and substitution of various metal ions (Jauhar, 2016).

Meanwhile, the research about the rheological properties of Cobalt Ferrite NanoFluids (CFFs) has been minimal, although these properties determine their application capacity. In the study of Steffen Thurm (Thurm \& Odenbach, 2003), the rheological properties of CFFs with the size dependence was investigated. Similar work was 
done by Mahesh Chand (Chand et al., 2013). The rheological properties of CoNi fluids were examined for both nanosphere and nanotube particles by T. Lopez-Lopez(López-López et al., 2012). The magnetoviscous properties of the amorphous magnetic fluids of Co-B particles were studied in comparison with $\mathrm{CoFe}_{2} \mathrm{O}_{4}$ at $1.8 \mathrm{wt} \%$ of magnetic particles (Yang et al., 2018).

As seen in the above studies, the influence of the parameters on CFFs needs to be investigated. Thus, in the current work, the rheological behavior of CFFs under an external magnetic field with various strengths were considered. The preparation of CFNPs by the precipitation method was tailored to get ferrimagnetic and superparamagnetic particles. The viscosity investigation was carried out for both fluids.

\section{METHODS}

\section{Materials}

Ferric chloride hexahydrate $\left(\mathrm{FeCl}_{3} \cdot 6 \mathrm{H}_{2} \mathrm{O}, \mathrm{AR}\right)$ and Cobalt chloride $\left(\mathrm{CoCl}_{2} \cdot 6 \mathrm{H}_{2} \mathrm{O}, \mathrm{AR}\right)$ are provided by BDH Chemicals Ltd. England; Sodium hydroxide $(\mathrm{NaOH})$ is supplied by Merck.

\section{Cobalt Ferrite Fluid Preparation}

In this study, CFNPs were prepared by the co-precipitation procedures as follows. The solutions of $1 \mathrm{M} \mathrm{FeCl} \mathrm{l}_{3}$ and $0.5 \mathrm{M}$ $\mathrm{CoCl}_{2}$ both in $1 \mathrm{M} \mathrm{HCl}$ were prepared as stock ones. The mixture of $20 \mathrm{ml}$ of the stock solutions with the molar ratio of $\mathrm{Fe}^{3+}$ to $\mathrm{Co}^{2+}$ as 2:1 was added into a reaction vessel containing $100 \mathrm{ml}$ of the $\mathrm{NaOH}$ solution with $\mathrm{pH}=14$. During the reaction of 2 hours, the stir rate of 650 rpmand the $\mathrm{pH}$ of 14 were remained. The reaction temperature was controlled to get two kinds of CFNPs with different magnetic properties: ferrimagnetism and superparamagnetism. The resulting precipitates were separated by a strong magnet and then washed manytimes with distilled water until the $\mathrm{pH}$ reached neutral and there was not chloride ion in the washed water. After that, the particles were dispersed in water at various weight fractions by ultrasonication for several hours until there was no visible precipitate found.

\section{Characterization of Cobalt Ferrite Fluids}

The structure of the CFNPs was characterized by X-ray diffraction (XRD) measurements, using a D8-AdvancBruker AXS diffractometer with $\mathrm{CuK} \alpha$ radiation $(\lambda=1.542 \AA)$ operating at $25^{\circ} \mathrm{C}$. The shape, size, and Particle Size Distribution (PSD) of CFNPs were calculated directly from Transmission Electron Microscopy (TEM) images with the instrument JEOL EM 1010. The magnetic characteristics, such as saturation magnetization and coercivity value of CFNPs were evaluated by a Vibrating Sample Magnetometer (VSM) MicroSense EZ9. The VMS measurements were carried out from 0 to $16 \mathrm{kOe}$ field and vice versa.

Nanoparticles weight fraction was estimated by a SETARAM ThermoGravimetric Analyzer (TGA). The measurements were taken under nitrogen gas with a heating rate of $15^{\circ} \mathrm{C} / \mathrm{min}$ from 25 to $600{ }^{\circ} \mathrm{C}$.

\section{Viscosity Measurement}

A HAAKE RotoVisco 1 Rheometer was used to measure the viscosity of cobalt ferrite nanofluids, either the presence or the absence of a magnetic field. The viscosity measurements were done at a temperature of $27 \pm 1{ }^{\circ} \mathrm{C}$. Since there was no precipitate observed in the fluid samples in stored days, the impact of agglomeration in the CFFs could be ignored in the viscosity results. 


\section{RESULTS}

\section{Cobalt Ferrite Fluid Characterizations}

The characterization of the prepared particles and corresponding fluids are given in table 1. The X-ray spectroscopies of the synthesized particles are presented in figure 1. At all the samples, the XRD results (figure 1) show the diffraction peaks (220), (311), (400), (422), (511), and (440) which are matched to the cubic spinel structure (JCPDS card no. 22-1086). The TEM analyses indicate that all the particles are spherical and their diameters vary in the range $2-20 \mathrm{~nm}$ (figure 2). The average particle sizes $\left(\mathrm{d}_{\mathrm{avg}}\right)$ of the samples are listed in table 1 .

Table 1: Characterizations of CFNPs

\begin{tabular}{|c|c|c|c|c|}
\hline Sample & $\begin{array}{c}\mathrm{C} \\
(\% \text { wt. }) \\
\end{array}$ & $\begin{array}{c}\mathbf{d}_{\text {avg }} \\
(\mathrm{nm})\end{array}$ & $\begin{array}{c}\mathrm{Ms} \\
(\mathrm{emu} / \mathrm{g})\end{array}$ & $\begin{array}{c}\mathrm{H}_{\mathrm{c}} \\
(\mathrm{Oe}) \\
\end{array}$ \\
\hline \multicolumn{5}{|l|}{ M1 } \\
\hline M1.1 & 1.0 & \multirow{3}{*}{10.7} & \multirow{3}{*}{61.6} & \multirow{3}{*}{205.7} \\
\hline M1.2 & 3.0 & & & \\
\hline M1.3 & 5.0 & & & \\
\hline \multicolumn{5}{|l|}{ M2 } \\
\hline M2.1 & 0.5 & \multirow{3}{*}{5.7} & \multirow{3}{*}{22.5} & \multirow{3}{*}{0} \\
\hline M2.2 & 1.0 & & & \\
\hline M2.3 & 5.0 & & & \\
\hline
\end{tabular}

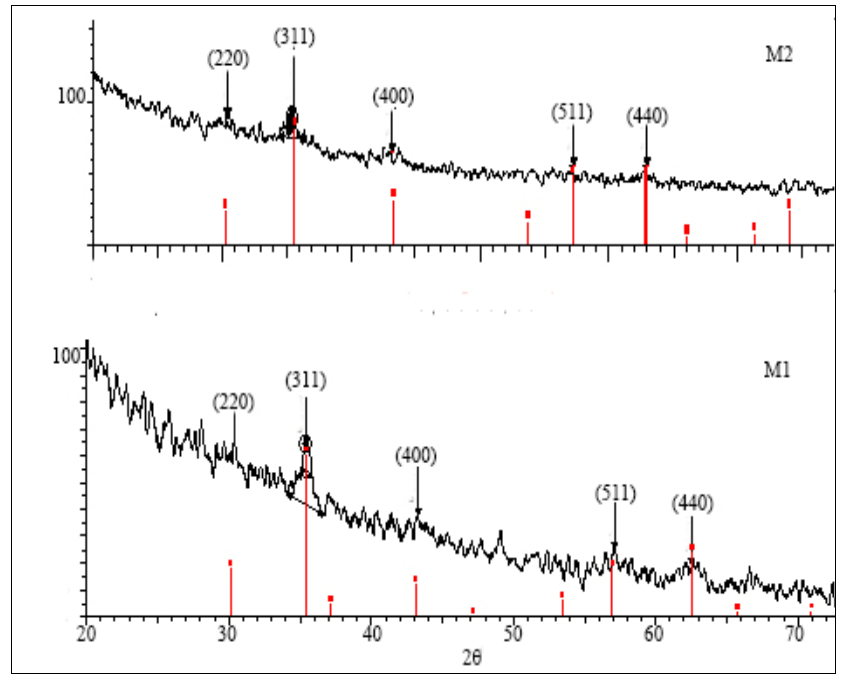

Figure 1: XRD Patterns of the Synthesized Particles.
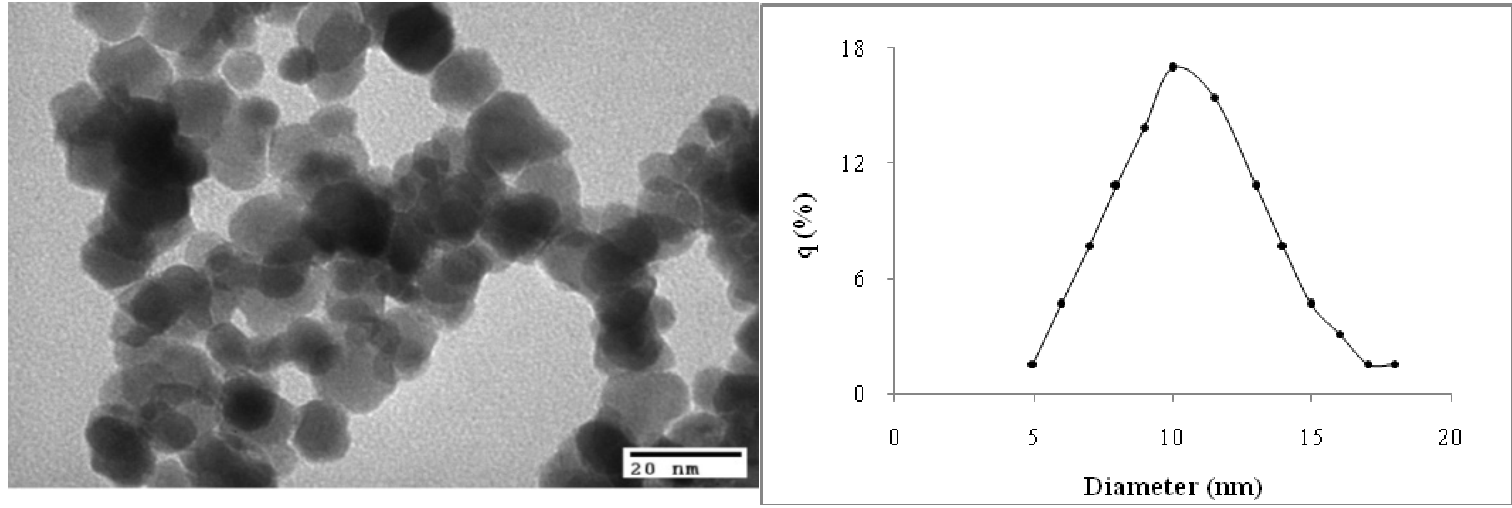

Figure 2: TEM Pictures and PSDs of CFNPs (M1: below, M2: Above). 
The room temperature magnetizations for the applied magnetic field of the prepared nanoparticles are presented in figure 3. The saturation magnetization (Ms) and coercivity $(\mathrm{Hc})$ are given in table 1 . The results demonstrate that the M2 particles show a typical property of superparamagnetic particles with $\mathrm{Hc}=0$. The M1 particles are ferrimagnetic ones with a saturation magnetization of $61.6 \mathrm{emu} / \mathrm{g}$ and $\mathrm{Hc}=205.7 \mathrm{Oe}$. The particle concentration, $\mathrm{C}$ (table 1) was calculated from TGA measurements. As seen in figure 4, the TGA curve of a typical fluid indicates one endothermic peak at $\sim 150{ }^{\circ} \mathrm{C}$, which may be attributed to the evaporation of water. The residual mass is considered as the amount of cobalt ferrite particles.

\section{Rheological Properties of Cobalt Ferrite Fluids}

A non-linear correlation between shear stress and shear rate is observed at all the CFFs (figure 5). The result indicates that the investigated fluids are non-Newton fluids. The viscosity of these fluids decreases as the shear rate is increased, showing the shear-thinning behavior. This behavior is found in the fluids under the magnetic field with various intensities. Figure 5 also shows both shear stress and viscosity are raised as the magnetic field is strengthened. The enhancement in viscosity depends on the shear rate. For example, sample M1.1, with the field intensity changed from 0 to $224 \mathrm{mT}$, the viscosity increases from 3.09 to $8.65 \mathrm{mPa} . \mathrm{s}\left(5.56 \mathrm{mPa}\right.$. s) at $12.9 \mathrm{~s}^{-1}$ and from 0.96 to $2.31 \mathrm{mPa} . \mathrm{s}(1.35 \mathrm{mPa}$. $\mathrm{s})$ at $100 \mathrm{~s}^{-1}$. The fielddependent viscosity was reported for iron oxide fluids (R.Y. Hong, 2007), (A. Amirimehr, 2012), (Rajnak et al., 2019) and for cobalt ferrite fluids (Chand et al., 2013). This behavior has been related to the arrangement of magnetic particles in chain-like or drop-like structures (Horng et al., 2001) along the direction of the external field, which is perpendicular to the direction of shear rate in our work. The new structures hinder the free rotation of particles, therefore, increasing viscosity. The higher the magnetic field strength, the stronger the interaction of the particle with the magnetic field and the higher viscosity is. A decrease of the relative viscosity of $\gamma-\mathrm{Fe}_{2} \mathrm{O}_{3}$ paraffin-based ferrofluid at the magnetic field above $100 \mathrm{kA} / \mathrm{m}$ was reported by Hosseini (Masoud Hosseini et al., 2010). This result is attributed to the phase separation in the ferrofluid. In our study, the different size distribution of nanoparticles causes the phase separation is not found (see figure 2).

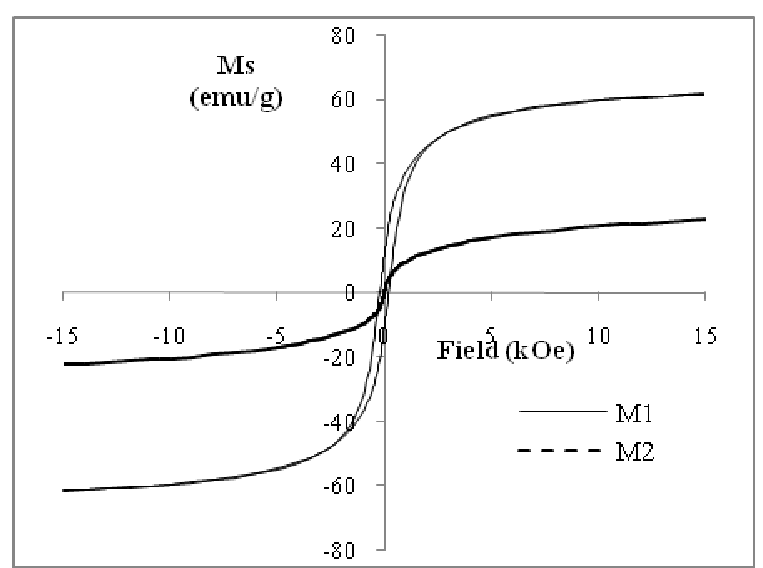

Figure 3: Magnetization Curves of CFNPs

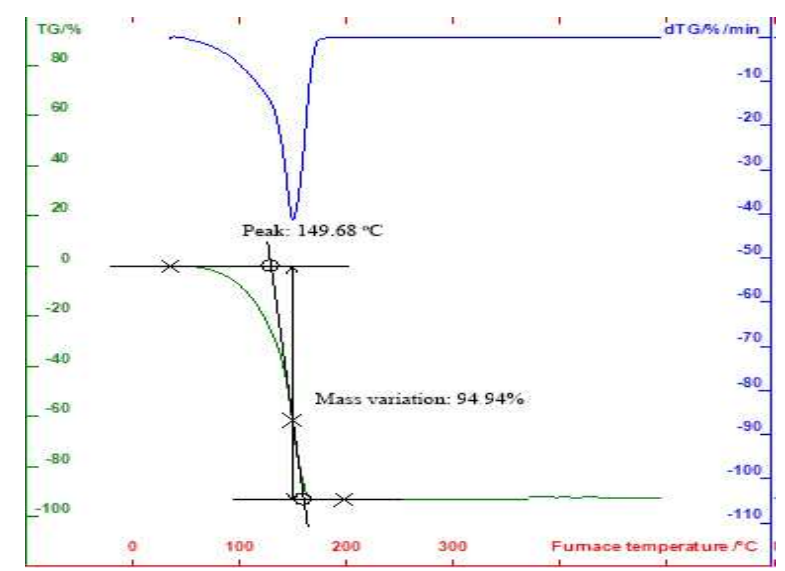

Figure 4: Weight Loss Curves of a Typical Nanofluid (M1.3).

At low shearing rates, the magnetically induced structures offer a significant obstruction of the flow leading to a high value of viscosity. When the shear rate is gradually raised, the structures begin to align themselves with the shear direction or break into smaller sizes. This structure change causes the viscosity reduction when the shear rate was increasing. At first, the viscosity rises owing to the domination of the formation of new structures hindering the flow. After that, the formation of structures reaches its ultimate level and only the effect of shear rate is active (see figure 5b). 
The field-dependent viscosity behaviors are observed in both kinds of CFFs. However, the enhancement of viscosity at the M2 sample is quite higher than the M1 sample (figure $5 \mathrm{~d}$ compared to figure $5 \mathrm{~b}$ ). This result indicates that the influence of magnetic fields on the viscosity in the M2 sample is more significant than that in the M1 sample. It should be noted that the average particle diameter of the M2 fluid is relatively smaller than the M1 (5.8 $\mathrm{nm}$ versus $10.7 \mathrm{~nm})$. The increase of viscosity with an increase of particle size that was found in some other nanofluids is not observed in our research. Therefore, we deduce that in the range of particle size from 2 to $20 \mathrm{~nm}$ of this study (see PSDs in figure 2), the number density of particles contributes to the field-dependent viscosity more than the particle size. The M2 fluid with the smaller particles has the lager density of particles, which enhances the magnetic interaction to form clusters and ultimately enhancing the viscosity. A similar result was also found in the work of Mahesh Chand (Chand et al., 2013).

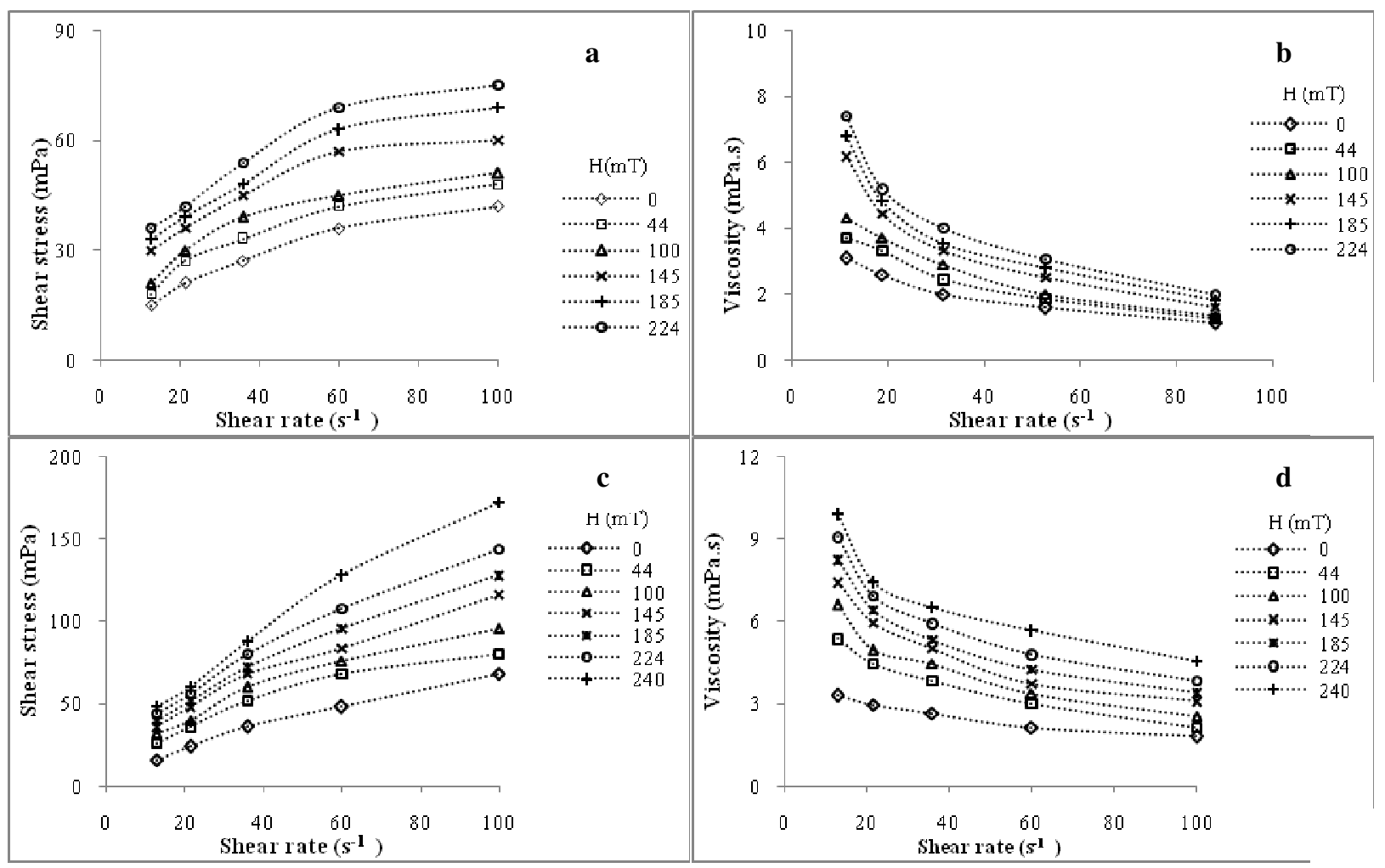

Figure 5: Shear Stress $(\tau)$ and Viscosity $(\eta)$ Plotted vs Shear Rate Dependence at Different Magnetic Field Intensities (PARTS a and b: Sample M1.1; Parts c and d: Samplem2.2).

The viscosity change owing to the external field application was quantified via the MagnetoViscous Effect (MVE) as follows

$$
M V E=\frac{\eta_{H}-\eta_{o}}{\eta_{o}}
$$

Where $\eta_{H}$ and $\eta_{0}$ are the viscosities of the CFFs at $\mathrm{H}$ and zero magnetic field intensities, respectively

The MVE dependence on the shear rate is performed in figure 6. As seen, the MVE shows a downward trend with the shear rate in both CFFsat all the magnetic field magnitudes. This tendency is quite understandable as the shear rate increases, the destruction degree of the structures which resist the flow also increases (López-López et al., 2012). As a result, the viscosity decreases. However, the difference in MVE values between the M1 and the M2 nanofluid is remarkable (figure $6 \mathrm{a}$ and $6 \mathrm{~b}$ ). For the M2 fluid, the MVE-magnetic field dependence is more potent. This result is 
explained by the higher number density of particles in the M2, as mentioned above. On the other hand, the ferrimagnetic particles in the M1 fluid are high magnetocrystalline anisotropy so that their magnetic moments are hardly aligned with the magnetic field (Bacri et al., 1995). Thus, the magnetic field dependent viscosity at these fluids is weaker.

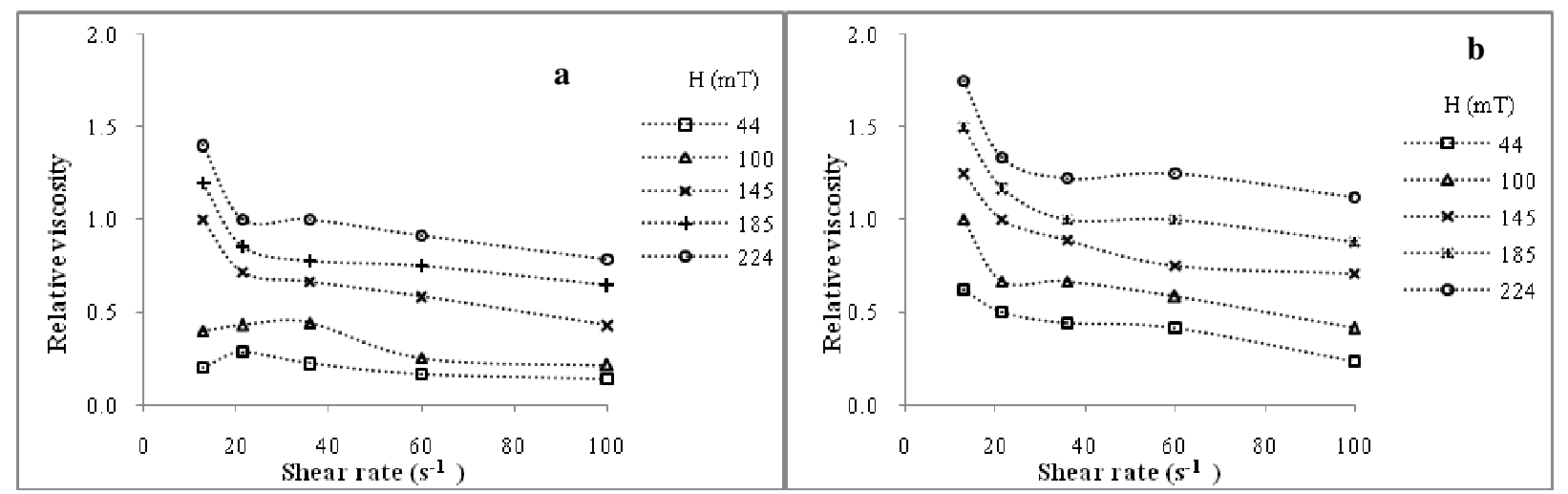

Figure 6: Relative Viscosity Plotted vs the Shear Rate Dependence under Different Intensities of the Magnetic Field (Parts a: Sample M1.1; Parts b: Sample M2.2).

The MVE values found in the current study are similar with the result (20\%) was reported by Rajnak for Cobalt ferrite fluids with volume fraction $1-2 \%$ at $\mathrm{H}=53 \mathrm{mT}$, shear rate $=20 \mathrm{~s}^{-1}$ (Rajnak et al., 2019). However, compared to the others, in which the $\mathrm{Fe}_{3} \mathrm{O}_{4} / \mathrm{H}_{2} \mathrm{O}$ ferrofluids were examed at the same investigated conditions, a somewhat lower degree in the magnitude of MVE is found. The MVE values of (Gu et al., 2013), (Malekzadeh et al., 2016) (Paul et al., 2016) (Yang et al., 2014) respectively were 1.6, 3, 5, và 7. This difference is attributed to the strong magnetocrystalline anisotropy (K1 $\approx 2 \times 106 \mathrm{erg} / \mathrm{cm} 3$ ) of Cobalt ferrite particles(Shahjuee, 2017), as explained above.

\section{Effect of Particle Concentration}

The viscosity as a function of particle concentration is presented in figure $7(\mathrm{a}, \mathrm{c})$. The viscosity seems to increase linearly with the particle weight fraction at all the magnetic field intensities. As mentioned above, with the presence of a magnetic field, the particles aggregate to form chain-like or drop-like structures along the direction of the magnetic field. This arrangement becomes more evident when the magnetic field increases and the particle concentration is higher. Thus, the viscosity is enhanced. These behaviors are observed for both M1 and M2 fluids. And as mentioned in the previous part, the difference in the viscosity enhancement between two fluids is attributed to the number density of particles.

For the M1 samples, the viscosity tends to a stable value at the shear rate of $100 \mathrm{~s}^{-1}$ (figure $6 \mathrm{~b}$ ). This behavior is related to the balance of the magnetic and mechanic effect on the free rotation of CFNPs in the shear flow. This trend is not observed in the M2 samples (figure $6 \mathrm{~d}$ ). This result is understood that the M2 viscosity will saturate at a shear rate higher than the M1. The values of the saturation shear rate are estimated by extrapolating the values of the shear rate at which the viscosities of the fluids with and without magnetic fields are equal. The values are $390 \mathrm{~s}^{-1}$ and $630 \mathrm{~s}^{-1}$ for the M1 and the M2 fluid, respectively. The previous researches (Zubarev \& Iskakova, 2004)(Odenbach, 2003) supposed that the large particles in the fluid determine the fluid viscosity under a magnetic field application. The MVE is created by the largest particles at the weak field and by the small ones at the strong field (Masoud Hosseini et al., 2010). This theory is a reasonable explanation for the fact that the saturation shear rate of the M2 fluid is higher than the M1 fluid. 


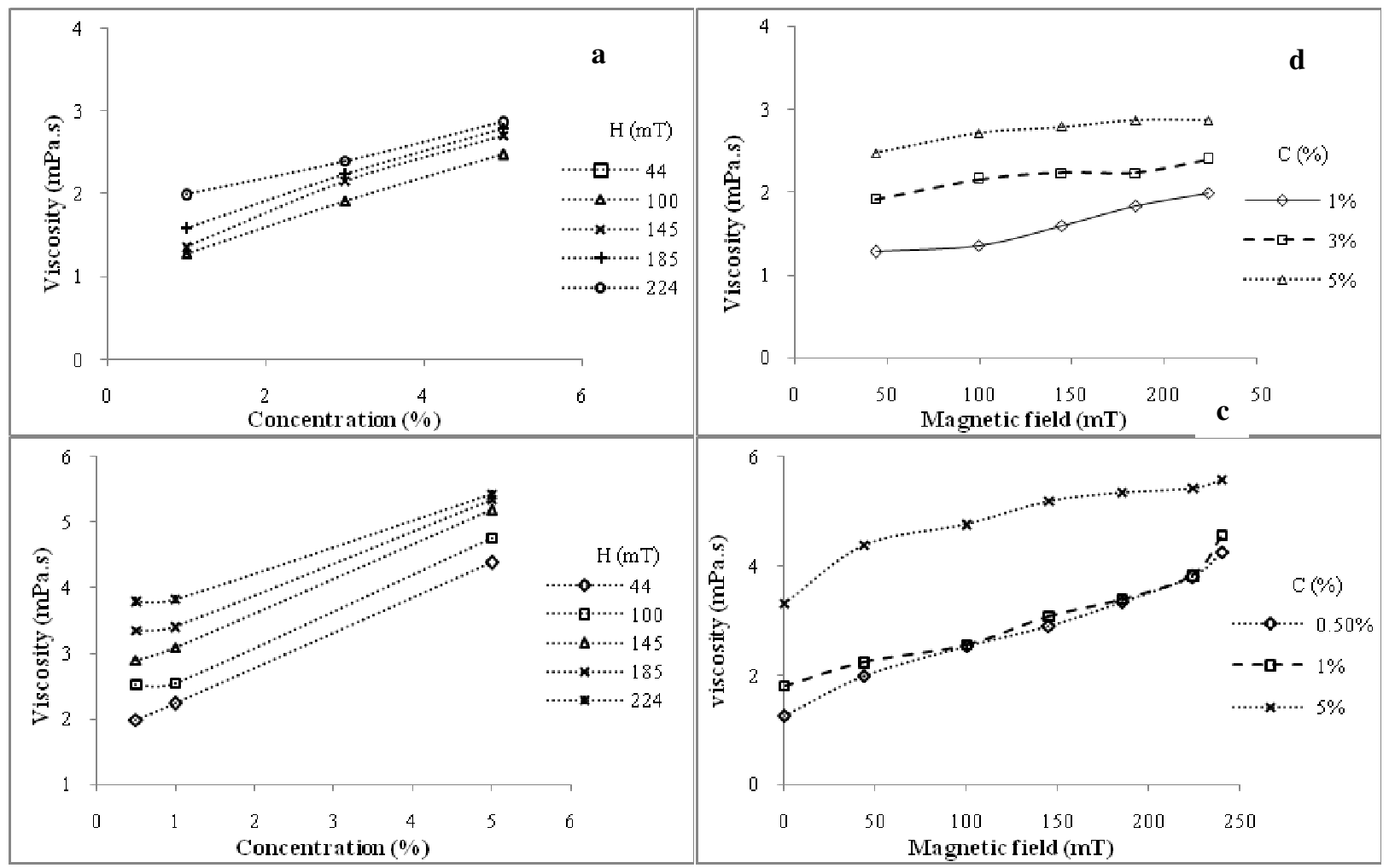

Figure 7: Viscosity $(\eta)$ Plotted vs Particle Concentration and Magnetic Field at Shear Rate $100 \mathrm{~s}^{-1}$ (Parts a and b: M1 Samples; Parts c and d: M2 Samples).

In figure 8, the MVE values of the fluids are compared at different concentrations. For the M2 fluids, the viscous effect increases as increasing the external magnetic field and decreases with increasing the particle concentration. The MVE is strongest at the lowest concentration of $0.5 \%$. This result can be attributed to the significant effect of the M2 fluid with concentration interaction without a magnetic field application. The lower the concentration, the less viscosity ( $\left.\eta_{\mathrm{o}}\right)$ is, which results in a higher MVE. These arguments also explain the opposite result in the M1 fluid, where the MVE increases (although not entirely apparent) as the concentration is increased.

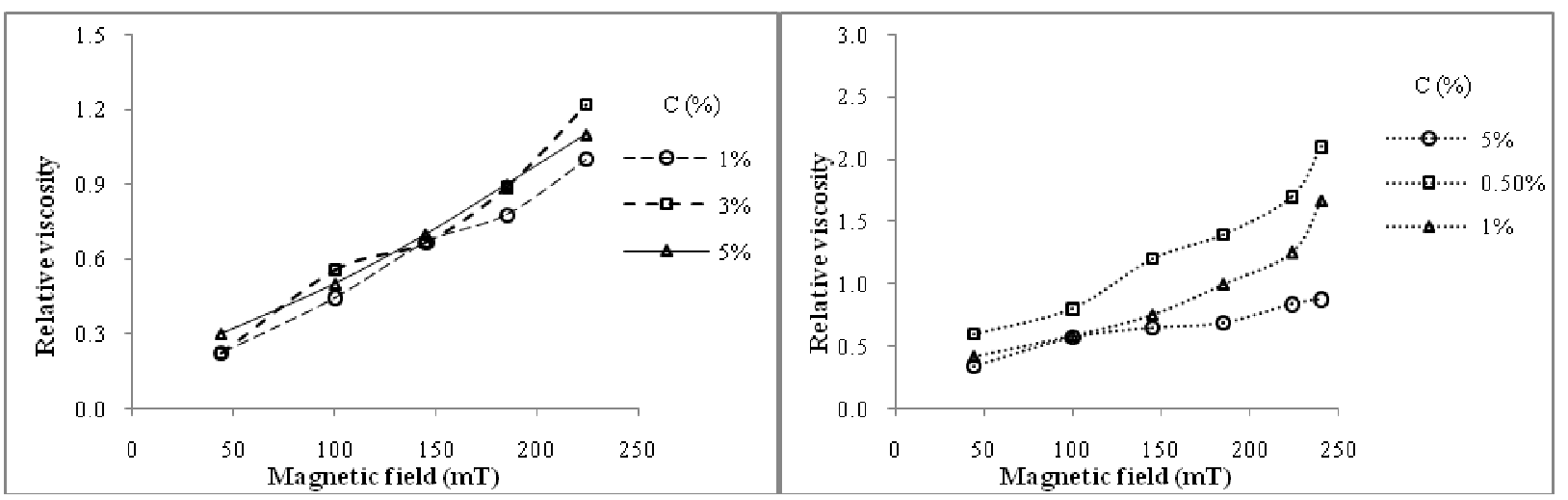

Figure 8: Relative Viscosity Shown with Increasing Magnetic Field for CFFs with Various Concentrations at Shear Rate $60 \mathrm{~s}^{-1}$ (Parts a: Sample M1; parts b: Sample M2).

\section{CONCLUSIONS}

The rheological properties of two kinds of cobalt ferrite nanofluids were studied. The influence of particle concentration on the magnetoviscous effect of the CFFs was investigated. The viscosity results are considered concerning the magnetic and 
structural properties. All the fluids show shear thinning behavior. The field-dependent viscosity of the fluids containing superparamagnetic particles is more significant as compared to the fluids of ferrimagnetic particles. The result is attributed to the higher number density of M2 particles in the fluids and the difference in magnetic properties. The viscosity of CFFs enhances with the increase the magnetic field owing to the changes in structure. This effect increases when the particle concentration is increased. These results are observed in both kinds of cobalt ferrite fluids. The magnetoviscous effect isincreased in a mono tone with the magnetic field and reversed with the shear rate. The obtained effects are somewhat lower than those of common iron oxide ferrofluids. The more substantial MVE in the nanofluids based on superparamagnetic particles is observed.

\section{ACKNOWLEDGEMENTS}

This research is funded by Vietnam National Foundation for Science and Technology Development (NAFOSTED) under grant number 104.06-2017.42.

\section{REFERENCES}

1. A. Amirimehr, A. Fazlali, E. G. (2012). Rheological properties of a NiFe3O4 paraffin-base ferrofluid. Proceedings of the 4th International Conference on Nanostructures, (pp. 1403-1406).

2. Amiri, S., \& Shokrollahi, H. (2013). The role of cobalt ferrite magnetic nanoparticles in medical science. Materials Science \& Engineering C, 33(1), (pp. 1-8).

3. Bacri, J. C., Perzynski, R., Shliomis, M. I., \& Burde, G. I. (1995). Negative-viscosity effect in a magnetic fluid. Physical Review Letters, 75(11), (pp. 2128-2131)

4. Chand, M., Kumar, S., Shankar, A., Porwal, R., \& Pant, R. P. (2013). The size induced effect on rheological properties of Coferrite based ferrofluid. Journal of Non-Crystalline Solids, 361(1), (pp. 38-42).

5. Horng, H. E., Hong, C. Y., Yang, S. Y., \& Yang, H. C. (2001). Novel properties and applications in magnetic fluids. Journal of Physics and Chemistry of Solids, 62(9-10), (pp. 1749-1764).

6. Jauhar, S., Kaur, J., Goyal, A., \& Singhal, S. (2016). Tuning the properties of cobalt ferrite: A road towards diverse applications. In RSC Advances, 6(100), (pp. 1-72).

7. Job, VICTOR M., and S. Rao Gunakala. "Unsteady MHD Free Convection Couette Flow between Two Vertical Permeable Plates in the Presence of Thermal Radiation Using Galerkin's Finite Element Method." International Journal of Mechanical Engineering 2.5 (2014): 99-110.

8. López-López, M. T., Gómez-Ramírez, A., Rodríguez-Arco, L., Durán, J. D. G., Iskakova, L., \& Zubarev, A. (2012). Colloids on the frontier of ferrofluids. Rheological properties. Langmuir, 28(15), (pp. 6232-6245).

9. Masoud Hosseini, S., Fazlali, A., Ghasemi, E., Ahmadi Moghaddam, H., \& Salehi, M. (2010). Rheological properties of a $\gamma-$ Fe2O3 paraffin-based ferrofluid. Journal of Magnetism and Magnetic Materials, 322(23), (pp. 3792-3796).

10. Nasrawi, Hayder Kraidi Rashid. "Natural Convection Heat Transfer inside an Inclined Square Enclosure Filled with Al2o3 Nanofluid in Presence of Pair of Discrete Heat Flux Sources in Bottom Wall." International Journal of Mechanical Engineering (IJME) 3.1 (2014): 35-46.

11. Odenbach, S. (2003). Ferrofluids - Magnetically controlled suspensions. Colloids and Surfaces A: Physicochemical and Engineering Aspects, 217(1-3), (pp. 171-178). 
12. R.Y. Hong, Z.Q. Ren, Y.P. Han, H.Z. Li, Y. Zheng, J. D. (2007). Rheological properties of water-based Fe3O4 ferrofluids. Chemical Engineering Science, 62, (pp. 5912-5924).

13. Rajnak, M., Wu, Z., Dolnik, B., Paulovicova, K., Tothova, J., Cimbala, R., Kurimsky, J., Kopcansky, P., Sunden, B., Wadso, L., \& Timko, M. (2019). Magnetic field effect on thermal, dielectric, and viscous properties of a transformer oil-based magnetic nanofluid. Energies, 12(23), (pp. 1-11)

14. Rashid, F., K. Dawood, and A. H. M. E. D. Hashim. "Maximizing of solar absorption by (TiO2-water) nanofluid with glass mixture." International Journal of Research in Engineering \& Technology 2 (2014): 87-90.

15. Rao, B. Madhushdhana, G. Viswanatha Reddy, and M. C. Raju. "Unsteady MHD mixed convection of a viscous double diffusive fluid over a vertical plate in porous medium with chemical reaction, Thermal radiation and joule heating." International Journal of Applied Mathematics \& Statistical Sciences (IJAMSS) 2.5 (2013): 93-116.

16. Sabah H. Sabeeh, Z. S. A. (2017). Preparation and Studying Magnetic Properties of Cobalt Ferrite (CoFe2O4) Material. International Journal of Sciencetific \& Engineering Research, 8(3).

17. Shahjuee, T., Morteza Masoudpanah, S., \& Mohammad Mirkazemi, S. (2017). Coprecipitation Synthesis of CoFe2O4 Nanoparticles for Hyperthermia. Journal of Ultrafine Grained and Nanostructured Materials, 50(2), (pp. 105-110)

18. Sharifi, I., Shokrollahi, H., \& Amiri, S. (2012). Ferrite-based magnetic nanofluids used in hyperthermia applications. Journal of Magnetism and Magnetic Materials, 324, (pp. 903-906).

19. Srinivasan, S. Y., Paknikar, K. M., Bodas, D., \& Gajbhiye, V. (2018). Applications of cobalt ferrite nanoparticles in biomedical nanotechnology. Nanomedicine, 13(10), (pp. 1221-1238).

20. Thurm, S., \& Odenbach, S. (2003). Particle size distribution as key parameter for the flow behavior of ferrofluids. Physics of Fluids, 15(6), (pp. 1658-1664).

21. Qashqaei, Amir, and Ramin Ghasemi Asl. "Numerical Modeling And Simulation Of Copper Oxide Nanofluids Used In Compact Heat Exchangers." International Journal of Mechanical Engineering, 4 (2), 18 (2015).

22. Yang, C., Yu, M., Zhao, S., Tian, Y., \& Bian, X. (2018). Magnetoviscous Property and Hyperthermia Effect of Amorphous Nanoparticle Aqueous Ferrofluids. Nanoscale Research Letters, 13.

23. Zubarev, A. Y., \& Iskakova, L. Y. (2004). To the theory of rheological properties of ferrofluids: Influence of drop-like aggregates. Physica A: Statistical Mechanics and Its Applications, 343(1-4), (pp. 65-80). 
